\title{
Oropharyngeal Cancer and Human Papilloma Virus: Counselling First Line Health Professionals
}

\author{
Francis Gilbert, MD', Matthieu J Guitton, PhD, FRAI ${ }^{1,2}$ and Nathalie Audet, MDCM, FRCSC ${ }^{1,3^{*}}$
}

${ }^{1}$ Department of Otolaryngology and Ophthalmology, Faculty of Medicine, Université Laval, Quebec City, Quebec, Canada ${ }^{2}$ CERVO Brain Research Center, Quebec City, Quebec, Canada

${ }^{3}$ Department of Surgery, Service of Otolaryngology-Head \& Neck Surgery, Centre Hospitalier Universitaire de QuébecUniversité Laval, Quebec City, Quebec, Canada

*Corresponding author: Nathalie Audet, MDCM, FRCSC, Department of Otolaryngology and Ophthalmology, Faculty of Medicine, Université Laval; Department of Surgery, Service of Otolaryngology -Head \& Neck Surgery, Centre Hospitalier Universitaire de Québec-Université Laval, Quebec City, Quebec, Canada. 1401 18e rue, Québec (Québec), GIJ 1Z4, Canada, Tel: +1-418-649-5821, Fax: +1-418-649-5585

\begin{abstract}
Introduction: In the last decade, the incidence of Human papilloma virus (HPV)-associated oropharyngeal cancer has increased and as high as $70 \%$ of all oropharyngeal cancers are caused by HPV. First line health professionals might feel uncomfortable discussing topics related to sexual habits. The objectives of this study are to assess the level of knowledge about HPV-associated oropharyngeal cancer amongst first line health professionals and to evaluate the impact of an educational intervention on communication with patients.
\end{abstract}

Method: Basic levels of knowledge of HPV-associated oropharyngeal cancer and ease to deal with patient were evaluated for general practitioners (GP), head and neck specialists (HNS), and nurses. Each category of professional was divided into two groups: Participants who receive a single HPV training, and a control group. The training was a onehour presentation addressing the management of HPV-associated oropharyngeal cancer. Participants filled questionnaires about general demographic information, general knowledge of oropharyngeal HPV, the ease to deal with HPV patients and evaluation of the educational intervention.

Results: The sample consisted of 122 participants consisting of first line health professionals. All groups presented a significant increase of their knowledge following the training session. Post-training nurses out performed control GPs. Nurses with less than 10 years of practice presented higher scores than more experienced nurses. For all categories, the training resulted in a significant increase of their perceived ease to discuss head and neck HPV with patients.

Conclusion: Continuous education on HPV-oropharyngeal cancer is needed for health professionals and it improves immediate knowledge and ease of discussion.

\section{Keywords}

Human papilloma virus, HPV, Oropharyngeal cancer, Counselling, Education

\section{sIntroduction}

Human papilloma virus (HPV) is the most frequent sexually transmitted infection. The virus is highly prevalent in the general population. It is estimated that in the USA, respectively $84.6 \%$ and $91.3 \%$ of sexually active women and men will contract HPV at a point or another in their life [1]. The virus will be spontaneously eliminated within 2 years post-infection in $90 \%$ of the cases [2]. The HPV might get reactivated in some people, leading to the development of tumors typically taking place either in the anogenital region (cervix, vulva, penis, or anus) or in the oropharynx. Overall, the relationship between HPV and anogenital cancers is well known in the general and medical population, notably thanks to massive vaccination campaigns in most Western countries [3]. However, the incidence of HPV-associated oropharyngeal cancer exceeded that of HPV-associated cervical cancer in the last decade [4]. Nowadays, as high as $70 \%$ of oropharyngeal cancers are caused by HPV [5]. In contrast to what is seen for anogenital cancers, the general population is considerably less aware of the association between HPV and oropharyngeal cancer. Furthermore, this is true not only for the general population,

Citation: Gilbert F, Guitton MJ, Audet N (2021) Oropharyngeal Cancer and Human Papilloma Virus: Counselling First Line Health Professionals. Res Rep Oral Maxillofac Surg 5:048. doi.org/10.23937/26433907/1710048

Accepted: February 08, 2021, 2020; Published: February 10, 2021

Copyright: (C) 2021 Gilbert F, et al. This is an open-access article distributed under the terms of the Creative Commons Attribution License, which permits unrestricted use, distribution, and reproduction in any medium, provided the original author and source are credited. 
but also amongst health professionals [6,7]. Indeed, a survey-based study performed at a middle-size US university demonstrated that only one third of first year medical students, and only $70 \%$ of fourth year medical students were aware of the link between HPV and oropharyngeal cancer [6]. Similarly, a recent Italian study showed that less than a fourth of nursing students were aware of this relationship [7].

When receiving an HPV-related cancer diagnosis, patients will come with numerous questions. In addition of coping with cancer and its consequences, patients must manage the emotional burden of being affected by a sexually transmitted infection [8]. Since HPV transmission occurs during oro-genital sexual intercourse, both patients and health professionals might feel uncomfortable discussing topics related to sexual habits. This is especially true for first line health professionals, for instance for general practitioners or when the initial reference is made to a head and neck specialist [810]. Obviously, the fact that health professionals might not be comfortable with the subject could represent a problem for patient counselling, and ultimately care in a context of patient empowerment. Thus, this study was designed to evaluate the current state of knowledge of first line health professionals on HPV-related oropharyngeal cancers. We then evaluated the potential of a single 1-hour training session to alleviate both potential lack of knowledge and unease to deal with the topic. Therefore, the first objective of this study was to assess the level of knowledge about HPV infection and cancer amongst general practitioners, nurses, and head and neck specialists. The second objective was to evaluate the feasibility and potential of an educational intervention directed at first line health professionals to improve communication with patients.

\section{Materials and Methods}

\section{Protocol}

Basic levels of knowledge of HPV-associated oropharyngeal cancer and ease to deal with patients were evaluated for the 3 study populations: General practitioners (GP), head and neck specialists (HNS) and nurses. Each study population was divided into two groups, a group of participants who received the 1 hour HPV training and a group who did not receive the HPV training. Head and neck surgeons were defined as otorhinolaryngologist or maxillofacial surgeons. Nurses were coming from intensive care units, surgery floor, outpatient's clinics, or were oncology pivot nurses. Participants were recruited from the medical staffs of urban and rural public hospitals of the Quebec province (Canada). Participants were contacted using a snowball sampling approach. Data were collected from November 2018 to June 2019. Prior to beginning the study, the protocol was approved by the institutional ethics committee of the " $\mathrm{CHU}$ de Québec-Université Laval" (approval number: 4397).

\section{HPV Training-Educational intervention}

In order to assess the effect of the exposition to HPV training on the knowledge of HPV induced oropharyngeal cancer, a specific educational intervention was developed. The selected format was a one-hour lecture-like presentation addressing seven aspects of management of HPV induced oropharyngeal cancer: Pathophysiology, transmission, prevention, vaccination, screening, treatment, and approach to the patient. The presentation was developed by an oncologic head and neck surgeon and a senior resident in otorhinolaryngology and head and neck surgery using the most recent evidence-based studies on HPV. Educational interventions were performed by the designers of the teaching material.

\section{Questionnaires}

Three questionnaires were used in this study (Annexe 1). A first questionnaire combined general demographic information (gender (male, female, other, undisclosed), age (20-35 years, 36-50 years, 51-65 years, 66 years + ), profession, field of practice, years of practice (0-5 years, 6-10 years, 11-15 years, 16 years +$))$ and an evaluation regarding the ease of the professional to deal with HPV patients. This second part of the first questionnaire was made of five 10-points Likert scale items. A second questionnaire was developed to evaluate the general knowledge of the participants of HPV-associated oropharyngeal cancer. This 10 -items questionnaire contained Likert scale-based items. Participants of the 1-hour HPV training groups were tested immediately after the educational intervention. Long-term retention was not evaluated in the present study. Finally, a third questionnaire (which was only used for participants of the HPV training groups) was developed to evaluate the educational intervention itself. This 7-items questionnaire contained four Likert scale-based items (Table 1) (What is your global appreciation of the HPV training? Did the teachers seem competent to teach you? Was the subject taught interesting? Will this training change your practice?). Participants from the control groups completed electronic versions of the questionnaires. Participants of the HPV training groups completed a paper-version of the questionnaires.

\section{Statistical analysis}

Statistical analyses were performed using Mann-Whitney U-tests. When applicable, results are presented as mean \pm SEM.

\section{Results}

\section{Characteristics of the sample}

The final sample consisted of 122 participants consisting of medical staff from hospitals of the Quebec province (Canada). No questionnaires were excluded from the sample. All participants identified themselves 
Table 1: Evaluation of the HPV training on 10-points Likert scale items.

\begin{tabular}{|l|l|l|}
\hline \multirow{2}{*}{ What is your global appreciation of the HPV training? } & & Training group \\
\hline & GNS & $9.26 \pm 0.17$ \\
\hline \multirow{2}{*}{ Did the teachers seem competent to teach you? } & Nurse & $9.7 \pm 0.21$ \\
\hline \multirow{2}{*}{ Was the subject taught interesting? } & GP & $9.93 \pm 0.05$ \\
\hline & HNS & $9.61 \pm 0.14$ \\
\hline Will this training change your practice? & Nurse & $9.9 \pm 0.1$ \\
\hline & GP & $10 \pm 0$ \\
\hline & HNS & $9.52 \pm 0.15$ \\
\hline & Nurse & $9.9 \pm 0.1$ \\
\hline & GP & $9.97 \pm 0.03$ \\
\hline & HNS & $8.96 \pm 0.28$ \\
\hline & Nurse & $8.9 \pm 0.43$ \\
\hline
\end{tabular}

GP: General practitioners, HNS: Head and neck specialists

Table 2: Repartition of the participants depending on their age and numbers of years of practice of the participants.

\begin{tabular}{|c|c|c|c|c|c|c|c|c|c|c|}
\hline & & \multirow[b]{2}{*}{$\mathbf{N}$} & \multicolumn{4}{|l|}{ Age } & \multicolumn{4}{|c|}{ Years of practice } \\
\hline & & & $25-35$ & $36-50$ & $51-65$ & $66+$ & $0-5$ & $6-10$ & $11-15$ & $16+$ \\
\hline \multirow[t]{2}{*}{ GP } & Control group & 21 & 15 & 4 & 2 & 0 & 18 & 0 & 1 & 1 \\
\hline & Training group & 23 & 11 & 5 & 7 & 0 & 12 & 0 & 2 & 9 \\
\hline \multirow[t]{2}{*}{ HNS } & Control group & 14 & 14 & 0 & 0 & 0 & 14 & 0 & 0 & 0 \\
\hline & Training group & 10 & 6 & 4 & 0 & 0 & 6 & 2 & 2 & 0 \\
\hline \multirow[t]{2}{*}{ Nurse } & Control group & 24 & 18 & 6 & 0 & 0 & 14 & 2 & 6 & 2 \\
\hline & Training group & 30 & 6 & 20 & 4 & 0 & 1 & 6 & 3 & 20 \\
\hline
\end{tabular}

GP: General practitioners, HNS: Head and neck specialists

Table 3: Responses evaluating the ease of health care professionals to discuss with patients about ORL and non-ORL forms of HPV-related tumors on a 10-Likert scale.

\begin{tabular}{|c|c|c|c|}
\hline & & Training group & Control group \\
\hline \multirow[t]{3}{*}{ Do you discuss of head and neck HPV issues with your patients? } & HNS & $6.1 \pm 0.8$ & $4.57 \pm 0.92$ \\
\hline & GP & $1.57 \pm 0.3$ & $1.76 \pm 0.56$ \\
\hline & Nurse & $1.67 \pm 0.46$ & $1.25 \pm 0.57$ \\
\hline \multirow{3}{*}{$\begin{array}{l}\text { Are you comfortable discussing head and neck HPV issues with } \\
\text { your patients? }\end{array}$} & HNS & $8.3 \pm 0.54$ & $6.07 \pm 0.69$ \\
\hline & GP & $5.17 \pm 0.51$ & $3.19 \pm 0.7$ \\
\hline & Nurse & $5.93 \pm 0.6$ & $2.8 \pm 0.66$ \\
\hline \multirow{3}{*}{$\begin{array}{l}\text { Do you discuss of non- head and neck HPV issues with you } \\
\text { patients? }\end{array}$} & HNS & $3.1 \pm 0.92$ & $3.21 \pm 0.87$ \\
\hline & GP & $7.96 \pm 0.41$ & $5.9 \pm 0.52$ \\
\hline & Nurse & $1.5 \pm 0.47$ & $3.08 \pm 0.71$ \\
\hline \multirow{3}{*}{$\begin{array}{l}\text { Are you comfortable discussing non- head and neck HPV issues } \\
\text { with your patients? }\end{array}$} & HNS & $4.8 \pm 0.7$ & $4.29 \pm 0.73$ \\
\hline & GP & $7.83 \pm 0.41$ & $7.38 \pm 0.44$ \\
\hline & Nurse & $4.93 \pm 0.66$ & $4.79 \pm 0.76$ \\
\hline \multirow{3}{*}{$\begin{array}{l}\text { Do you consider it embarrassing for a health professional to } \\
\text { discuss HPV? }\end{array}$} & HNS & $3.8 \pm 0.93$ & $1.5 \pm 0.66$ \\
\hline & GP & $1.13 \pm 0.39$ & $0.57 \pm 0.19$ \\
\hline & Nurse & $2.4 \pm 0.56$ & $1.92 \pm 0.55$ \\
\hline
\end{tabular}

to one gender. The sample consisted of 44 GPs (control group: 21 (6 males, 15 females), HPV training group: 23 (23 females)), 24 HNSs (control group: 14 (6 males, 8 females), HPV training group: 10 (6 males, 4 females)), and 54 nurses (control group: 24 (3 males, 21 females),
HPV training group: 30 (30 females)). The repartitions of the participants for each of the subgroups depending on their age and number of years in practice are provided in Table 2. Our population consisted mainly of health care professionals working in a university hospital con- 
text (GP control group: 62\%.; GP HPV training: $87 \%$; HNS control group: $100 \%$; HNS HPV training group: $80 \%$; nurses control group: 79\%; nurses HPV training group: 93\%).

\section{Ease to deal with HPV-related issues}

Responses evaluating the ease of health care professionals to discuss with patients about head and neck and non-head and neck forms of HPV-related tumors are summarized in Table 3. There is no difference between GPs and nurses regarding the ease of discussion of head and neck HPV infections with patients $(p=0.2$ and $p=0.24$ for control and HPV training groups, respectively). Head and neck surgeons were discussing significantly more frequently this topic with their patients when compared to the other 2 groups $(p<0.05$ between HNS and GP control groups, $p<0.001$ between HNS and nurse control groups, $p<0.001$ between HNS and GP HPV training groups, and $p<0.001$ between HNS and nurse HPV training groups).

A similar pattern was evidenced for the perceived ease to discuss head and neck HPV infections with patients. There were with no significant differences observed between GPs and nurses ( $p=0.67$ and $p=0.17$ for control and HPV training groups, respectively). Both GPs and nurses were significantly less comfortable than HNSs when discussing head and neck HPV infections ( $p$ $<0.01$ between HNS and GP control groups; $p<0.01$ between HNS and nurse control groups; $p<0.01$ between HNS and GP HPV training groups, and $p=0.052$ between HNS and nurse HPV training groups).

General practitioners significantly discussed more

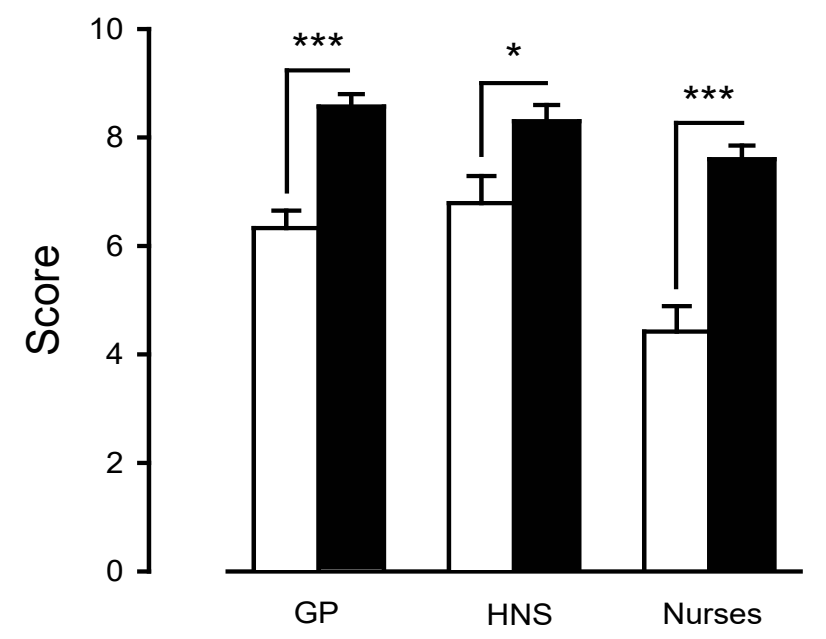

Figure 1: Control and post-training knowledge scores. This figure presents the control (white) and post-training (black) scores on the oropharyngeal HPV cancer knowledge questionnaire (minimal score: 0 , maximal score: 10 ), depending on the profession of the participants (GP: general practitioners, HNS: head and neck surgeons, nurses). Results were presented as mean $\pm \operatorname{SEM}\left({ }^{*} p<0.05,{ }^{* * *} p<\right.$ 0.001).

GP: General practitioners, HNS: Head and neck specialists frequently non head and neck HPV infection than both HNS and nurses $(p<0.05$ between GP and HNS control group, $p<0.001$ between GP and HNS HPV training group, $p<0.01$ between GP and nurse control group, $p<0.001$ between GP and nurse HPV training group). General practitioners were significantly more at ease to discuss non head and neck HPV infection than both HNS and nurses ( $p<0.01$ between GP and HNS control group, $p<0.001$ between GP and HNS HPV training group, $p<$ 0.05 between GP and nurse control group, $p<0.01$ between GP and nurse HPV training). For all professional categories, the HPV training resulted in a significant increase of their perceived ease to discuss head and neck HPV infections with patients ( $p<0.05$ for GPs; $p<0.05$ for HNSs; and $p<0.05$ for nurses).

\section{Pre-and post-training knowledge scores}

Initial scores were comparable for GP (score: 6.33 \pm 0.32 ) and HNS (score: $6.79 \pm 0.5$, no significantly different, $p=0.19$ ) but were significantly lower for nurses (4.42 $\pm 0.47, p<0.01$ from GP, and $p<0.01$ from HNS, Figure 1). All groups presented a significant increase of the immediate knowledge score following the training session (GP, score: $8.57 \pm 0.23$, different from related control group at $p<0.001$; HNS, score: $8.3 \pm 0.3$, different from related control group $p<0.05$; nurses, score: $7.6 \pm 0.25$, different from at the related control group at $p<0.001)$. Post-training nurses got significantly higher scores than untrained GPs $(p<0.01)$. Despite the limited size of some of the age groups, a trend was observed between the initial and post-training scores of nurses as a function of their experience (Figure 2). Nurses with less than 10 years of practice presented higher knowledge

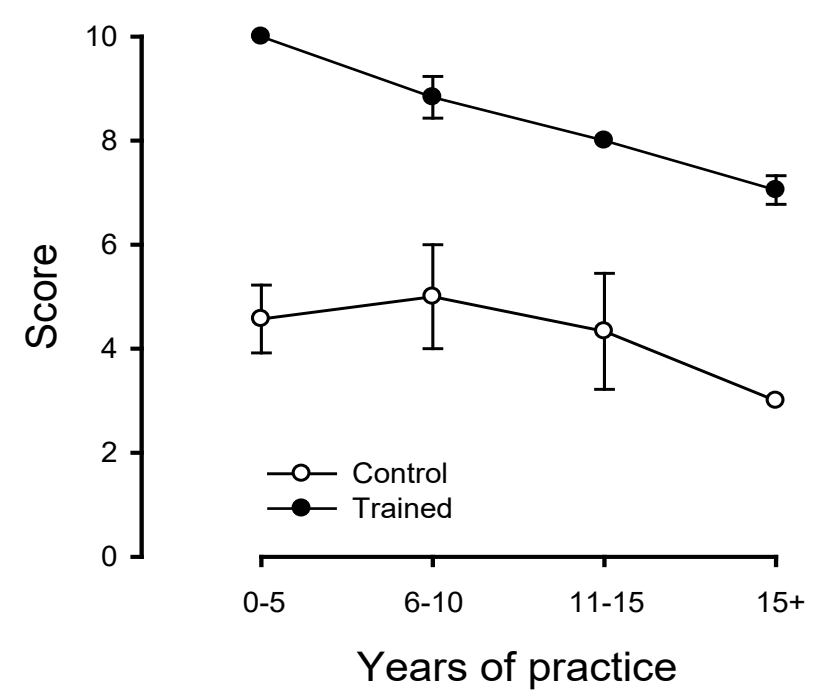

Figure 2: Knowledge score for nurses as a function of the years of practice.

This figure presents the scores on the oropharyngeal HPV cancer knowledge questionnaire (minimal score: 0 , maximal score: 10) for nurses as a function of the number of years of practice, for control (white dots) and participants receiving the HPV training (black dots). Results were presented as mean \pm SEM. 
scores than more experienced nurses. Furthermore, HPV training-related improvement of the knowledge score was less marked for nurses as working experience increased.

\section{Evaluation of the educational intervention}

Evaluations of the one-hour lecture on HPV-associated oropharyngeal cancer demonstrated high scores for the overall evaluation of the training session $(9.65$ \pm 0.08 on 10 , max: Nurses, $9.93 \pm 0.05$; min: GPs, $9.26 \pm$ $0.17)$, for the evaluation of the teachers $(9.84 \pm 0.06$ on 10; max: Nurses, 10 ; min: GPs, $9.61 \pm 0.14)$, or for the pertinence of the presented material $(9.79 \pm 0.06$; max: Nurses, $9.97 \pm 0.03$; min: GPs, $9.52 \pm 0.15)$. Whichever their group, attendants strongly expressed that the information they received during the training session will modify their future practice $(9.16 \pm 0.18$, max: Nurses, $9.4 \pm 0.27$; min: HNSs, $8.9 \pm 0.43$, not statistically different from Nurses, $p=0.12$, Table 1 ).

\section{Discussion}

Our study showed that HPV-associated oropharyngeal cancer knowledge is not optimal in first line health professionals, particularly for GPs and nurses. Furthermore, a 1-hour HPV training session resulted in a significant increase of the HPV-associated oropharyngeal cancer knowledge and of the perceived ease to discuss head and neck HPV infections with patients for all 3 professional categories. Human papilloma virus is a sexually transmitted infection that should concern all medical specialties, ranging from gynecology to otorhinolaryngology, from pediatric to oncologic patients $[9,10]$. Medical staff is the primary source of information for patients [11]. Doctors and nurses are considered the most trustworthy source of information according to a recent Canadian adult survey [12].

Human papilloma virus related to the head and neck site is seldom discussed as part of HPV related health issues. The first survey of HPV providers' vaccination practices and HPV knowledge in Canada since the introduction of school-based vaccination programs published in 2019 asked which potential consequences/benefit did the physicians counsel their patients about with respect to vaccination [12]. Although more Canadians are diagnosed with oropharyngeal cancer secondary to HPV infection than with cervical cancer [4] oropharyngeal cancer was not addressed by this survey. This reflects reality.

Despite being well documented, the link between HPV and oropharyngeal cancer is known enough by the general population, and to a certain extent by health workers. This was confirmed in this study. Cancer caused by HPV has been mainly associated with cervical cancer. The FDA recommended routine vaccination focused solely on female population until 2011. This led to a feminization of HPV awareness centered upon cervical cancer [8]. However, awareness of oro- pharyngeal cancer caused by HPV was left behind. This is mainly due to the fact that HPV induced oropharyngeal cancer is more prevalent in males. The absence of a screening method and pre-clinical endpoint make it difficult to establish a clear link between vaccination and oropharyngeal cancer prevention [13]. While the baseline knowledge of HPV-associated oropharyngeal cancer was initially not optimal for all categories of health professionals evaluated in this study, all of them showed a significant improvement following the 1-hour training session. Furthermore, all the participants received the training extremely positively, which suggest that this type of educational approach could represent a valid teaching strategy for continuous education on this topic. This reinforces the necessity to improve head and neck HPV awareness for all categories of healthcare professionals. As the goal of this study was to demonstrate feasibility - especially regarding the capacity to reach various categories of first line health professionals both in urban and rural settings - rather than developing a systematic educational program, long-term retention of the knowledge was not assessed. According to a recent Canadian adult survey, medical doctors and nurses are considered the most trustworthy source of information on HPV [12]. Dodd, et al. identified two main factors why discussing HPV related health issues with patient is difficult for head and neck cancer health professionals: Their lack of knowledge, and their reluctance to discuss sexuality with patients [14]. Our study showed that baseline knowledge was better for medical doctors than nurses. However, post-training nurses outperformed control GPs. As the first-line interlocutors of most patients, nurses play a critical role in counselling. Overall, the levels of knowledge on HPV-associated oropharyngeal cancers of the control nurses were very low. From a practical perspective, medical doctors are often contributing to the informal continuous training of nurses. Yet, the fact that medical doctors themselves were not displaying high degree of knowledge nor- and more problematically-high degree of comfort to discuss these topics with patients argues against an optimal inter-professional transmission of knowledge and best practices. Therefore, this emphasises even more the importance of offering such type of HPV-related specific training to nurses, in addition of medical doctors.

Interestingly, younger nurses displayed better knowledge than more experienced nurses. Although that could seem counter-intuitive at first, their good knowledge about HPV could be explained by public health determinants. Indeed, nurses who recently entered in practice belonged to the first female cohort in Canada to systematically receive HPV vaccine. Indeed, between 2007 and 2010, all Canadian provinces implanted HPV vaccination for young girls in the public vaccination program [15]. Despite the recent debates on vaccination emphasise the need for affirmative actions from the medical teaching community [16], HPV vaccination 
is the prevalent way to fight against the occurrence of HPV-related health issues $[17,18]$. Therefore, this early life exposition to HPV campaigns is likely to explain, at least partially, the over-performance of younger nurses compared to more experienced ones. Another contributing factor could be the addition of HPV-specific sections in initial professional curricula.

Due notably to anti-vaccination movements, ongoing vaccination campaigns are facing constant challenges [16]. The importance of the dynamic of the patient-doctor relationship is central in the decision-making process of the patient in order for the patient to submit to vaccination, and should not be underestimated. A recent national survey of Canadian adults on HPV demonstrated that the most important barrier to HPV vaccination was to not have a recommendation from a doctor [12]. In this context, our results might be worrisome, as they suggest that without specific training, doctors (either GPs or HNSs) mastery of the subject is not optimal-thus emphasizing the need to develop continuous medical education material on this topic.

Our study presents a few limitations. A potential bias is our sample of control and study population. The study group had a strong prevalence of clinicians working in a university hospital context. Thus, our study is likely to underestimate the real magnitude of lack of knowledge in community primary care units. Participants in the control group have agreed to answer the questionnaires and thus, might have had more interest regarding HPV-related topics, or more broadly, in continuous medical education in general. The second limit in this study is the absence of evaluation of long-term knowledge retention. Testing was performed directly after the training session. Given the specific objectives of this study and the lack of information regarding feasibility, such an evaluation was not added in the design in order to avoid facing dropout problems that would invalidate the study. Thus, we selected an immediate evaluation strategy over long-term measures. Nonetheless, and despite the very positive results on immediate retention, long-term knowledge retention will have to be evaluated in future studies.

Although the management of HPV-positive patients remains highly compartmentalize, this study clearly reinforces the notion that interdisciplinarity and inter-speciality collaboration is central. Medical specialists dealing with head and neck issues should take advantage of the work already done in other specialties. This longtime experience of other speciality translates not just in their individual expertise, but also in the structuring of their continuous education. For instance, gynaecologists have well-developed teaching and counselling tools, that other specialities would benefit to use. Beside its specific focus on HPV oropharyngeal cancers, this study could - both for its methodology and conclusions on the need of cross-disciplinary talk-contribute to a larger scale debate on interdisciplinarity in continuous medical education, for the greatest good of the population we have the care for.

\section{Conclusion}

This study evidenced the fact that basic knowledge of first line health professionals concerning HPV-associated oropharyngeal cancer is not what it should be. In addition to initial education, continuous education on HPV-oropharyngeal cancer is needed for first line health professionals, particularly for nurses, and to lesser extend general practitioners. A single 1-hour HPV training session with health professionals resulted in a significant increase of their immediate knowledge and perceived ease to discuss head and neck HPV infections with patients. Initiatives from the medical profession must be taken to improve this issue.

\section{Funding}

None.

\section{Conflict of Interest}

None.

\section{Code Availability}

Not applicable.

\section{Availability of Data and Material}

The data that support the findings of this study are available from the corresponding author, [NA], upon reasonable request.

\section{References}

1. Chesson HW, Dunne EF, Hariri S, Markowitz LE (2014) The estimated lifetime probability of acquiring human papillomavirus in the United States. Sex Transm Dis 41: 660-664.

2. Gravitt PE (2011) The known unknowns of HPV natural history. J Clin Invest 121: 4593-4599.

3. Brotherton JML, Bloem PM (2018) Population-based HPV vaccination programmes are safe and effective: 2017 update and the impetus for achieving better global coverage. Best Pract Res Clin Obstet Gynaecol 47: 42-58.

4. Statistique Canada (2016) VPH related cancer, IOP.

5. Van Dyne AE, Henley J, Sariya M, Cheryll CT, Markowitz LE, et al. (2018) Trends in Human Papillomavirus-Associated Cancers - United States, 1999-2015. Morb Mortal Wkly Rep 67: 918-924.

6. Evans L, Matley E, Oberbillig M, Margetts E, Darrow L (2020) HPV knowledge and attitudes among medical and professional students at a Nevada University: A focus on oropharyngeal cancer and mandating the vaccine. J Cancer E 35: 774-781.

7. Pelullo CP, Esposito MR, DiGiuseppe G (2019) Human Papillomavirus infection and vaccination: Knowledge and attitudes among nursing students in Italy. Int J Environ Res Public Health16: 1770.

8. Daley E, DeBate R, Dodd V, Dyer K, Fuhrmann H, et al. (2011) Exploring awareness, attitudes, and perceived role among oral health providers regarding HPV-related oral cancers. J Public Health Dent 71: 136-142. 
9. Kahn JA, Rosenthal SL, Tissot AM, Bernstein DI, Wetze C, et al. (2007) Factors influencing pediatricians' intention to recommend human papillomavirus vaccines. Ambul $\mathrm{Pe}$ diatr 7: 367-373.

10. Riedesel JM, Rosenthal SL, Zimet GD, Bernstein DI, Huang B, et al. (2005) Attitudes about human papillomavirus vaccine among family physicians. J Pediatr Adolesc Gynecol 18: 391-398.

11. Baxi SS, Shuman AG, Corner GW, Shuk E, Sherman EJ, et al. (2013) Sharing a diagnosis of HPV related head and neck cancer: The emotions, the confusion, and what patients want to know. Head Neck 35: 1534-1541.

12. Steben M, Durand N, Guichon JR, Greenwald ZR, McFaul S, et al. (2019) A National Survey of Canadian Adults on HPV: Knowledge, Attitudes, and Barriers to the HPV Vaccine. J Obstet Gynaecol Can 41: 1125-1133.
13. D'Souza G, Dempsey A (2011) The role of HPV in head and neck cancer and review of the HPV vaccine. Pred Med 53: S5-S11.

14. Dodd RH, Forster AS, Waller Jo, Marlow LAV (2017) Discussing HPV with oropharyngeal cancer patients: A cross-sectional survey of attitudes in health professionals. Oral Oncol 68: 67-73.

15. Canadian Immunization Committee (2014) Recommendations for Human Papillomavirus Immunization Programs. Ottawa: Public Health Agency of Canada, IOP.

16. Bloom DE, Fan VY, Sevila JP (2018) The broad socioeconomic benefits of vaccination. Sci Transl Med 16: 441

17. Barnabas RV (2012) Measuring the benefits of HPV vaccination. SciTransl Med 4: 148-149.

18. De Sanjose S, Delany-Moretlwe S (2019) HPV vaccine can be the hallmark of cancer prevention. Lancet 394. 